\title{
Cervical spinal cord compression in infants with achondroplasia: should neuroimaging be routine?
}

\author{
Victoria R. Sanders, M.S. ${ }^{1}$, Stephen H. Sheldon, D.O. ${ }^{2}$ and Joel Charrow, M.D. ${ }^{3}$
}

Purpose: To examine results of magnetic resonance imaging (MRI), polysomnograms (PSG), and patient outcomes in patients with achondroplasia in light of recent screening recommendations for infants with achondroplasia.

Methods: We reviewed medical records of 49 patients with achondroplasia followed at our institution between September 1997 and January 2017, including physical exams, MRIs, PSGs (when available), and surgical histories. Appropriate PSG data were available for 39 of these patients.

Results: Twenty-seven of 49 patients had cervical cord compression on MRI, and 20 of those patients required surgery. Central apnea was detected in $2 / 23$ patients with cervical cord compression in whom PSG data was available. Physical exam revealed depressed deep-tendon reflexes in two patients with cord compression and one patient without cord compression. Besides hypotonia in some, the neurological exams of these patients were unremarkable.

Conclusions: Cervical cord compression is a common occurrence in infants with achondroplasia and necessitates surgical intervention in some patients. Physical exam and PSG are poor predictors of the presence of cord compression or the need for surgery. All infants with achondroplasia should have MRIs of the craniocervical junction in the first 6 months of life.

Genetics in Medicine (2019) 21:459-463; https://doi.org/10.1038/s41436018-0070-0

Keywords: Foramen magnum stenosis; Cervical cord compression; Sudden death; Achondroplasia; Central apnea

\section{INTRODUCTION}

Achondroplasia (OMIM 100800) is the most common disproportionate short stature skeletal dysplasia with an incidence of approximately 1 in 26,000 (ref. ${ }^{1}$ ). Achondroplasia results in disproportionate short stature, macrocephaly, and increased rates of obstructive and central sleep apnea as well as foramen magnum stenosis. ${ }^{2-4}$ Infants and children under 4 years of age with achondroplasia are at increased risk of sudden death, and autopsy studies have revealed that sudden death was frequently attributable to lower brainstem or upper cervical spinal cord compression. ${ }^{3,5-7}$ Additionally, infants and young children have experienced apnea and acute life-threatening events; ${ }^{6,8-10}$ hemiparesis, paraparesis, or quadriparesis; $;{ }^{911}$ and new neurological impairment following minor trauma. ${ }^{12}$

Prior to the application of magnetic resonance imaging (MRI) to evaluate the craniocervical junction of infants with achondroplasia, there were no reliable methods to evaluate infants with achondroplasia to determine who might be at risk of injury or sudden death. Computed tomography (CT) scans can accurately measure the size of the foramen magnum but cannot predict the effect on the spinal cord. ${ }^{13}$ Somatosensory evoked potentials (SEPs) proved unreliable for the detection of cord compression (author's personal experience). ${ }^{14}$ As MRI became available in the 1980s it began to be used to screen infants for craniocervical compression. ${ }^{15,16}$ MRI is superior to $\mathrm{CT}$ in the evaluation of the craniocervical junction of patients with achondroplasia because MRI not only provides comparable measurements at the foramen magnum as compared with CT scans,${ }^{17}$ but also provides superior imaging of the brainstem and cervical cord, including cord compression and T2 signal changes, and visualizes posterior fossa structures, without ionizing radiation or exposure to contrast material. ${ }^{15,16}$ Multiple authors have recommended MRI screening of the craniocervical junction for all infants with achondroplasia. ${ }^{10,18,19}$ In addition, the American Academy of Pediatrics (AAP) guidelines on health supervision for children with achondroplasia (first published in 1995 (ref. ${ }^{20}$; updated in 2005 [ref. ${ }^{21}$ ]) recommend screening all infants with achondroplasia by CT or MRI. ${ }^{21}$ This recommendation has been recently challenged, ${ }^{22}$ and a debate in the literature has ensued. ${ }^{22-25}$ We therefore undertook this review of all patients with achondroplasia treated at our institution from September 1997 to January 2017 to examine the results of MRIs and patient outcomes.

\section{MATERIALS AND METHODS}

It has been our practice to obtain an MRI of the craniocervical junction for all patients with achondroplasia within the first 6 months of life, or as soon as possible if they are not seen

${ }^{1}$ Division of Genetics, Birth Defects and Metabolism, Ann \& Robert H. Lurie Children's Hospital of Chicago, Chicago, Illinois, USA; ${ }^{2}$ Director, Sleep Medicine Center, Ann \& Robert H. Lurie Children's Hospital of Chicago, Professor of Pediatrics \& Neurology, Feinberg School of Medicine, Northwestern University, Evanston, Illinois, USA; ${ }^{3}$ Division of Genetics, Birth Defects and Metabolism, Ann \& Robert H. Lurie Children's Hospital of Chicago, Professor of Pediatrics, Feinberg School of Medicine, Northwestern University, Evanston, Illinois, USA. Correspondence: Victoria R. Sanders (vsanders@luriechildrens.org) 
until after 6 months of age. We reviewed the electronic medical records and/or paper charts for all patients with achondroplasia first seen in our Skeletal Dysplasia Clinic between September 1997 and January 2017, for a total of 61 patients. Specifically, for each patient, in addition to demographic data, we recorded the dates and findings of the initial physical exams, MRIs, surgeries (if performed), and polysomnogram (PSG) results. Eight patients were excluded from our analysis because they were initially followed elsewhere and we do not have complete medical records from the outside hospitals available for review. Two patients were lost to follow-up after the initial visit and so were excluded. Two additional patients were also excluded because we did not have records of physical exam findings until 2 to 3 years of age. Eight patients did not have physical exam data available for review prior to their brain MRI. However, five of those patients had physical exams within a month of having an MRI, one patient was seen within 2 months, and two patients were seen within 3 months of having their initial MRI and so were included in this analysis. Twelve patients were therefore excluded in total, leaving 49 patients for our analysis. Seven additional patients were excluded from the PSG data because we did not have PSG results available in our records, and three other patients were excluded from the PSG data because PSGs were performed after the patients had posterior fossa decompression surgery, leaving 39 patients with PSG data available.

This study was exempted by the Institutional Review Board of the Ann and Robert H. Lurie Children's Hospital of Chicago.

\section{Case reports}

Patient 1 was initially seen in our clinic at 3 weeks of life. Physical exam was consistent with a diagnosis of achondroplasia. Neurological exam noted $1+$ reflexes and hypotonia and was otherwise normal. Medical history was unremarkable. We referred this patient for an MRI of the craniocervical junction per our normal practice. However, prior to the MRI being obtained, this patient experienced cardiorespiratory arrest exactly 8 days after her initial evaluation in our clinic. The patient was found unresponsive by her mother in her car seat. The mother started chest compressions prior to the arrival of emergency services. She was intubated at an outside hospital and transferred to our hospital. On arrival, she became appropriately responsive, with no reflexes elicited in the lower extremities. She was extubated, and an MRI of the brain was emergently obtained, revealing severe narrowing of the foramen magnum with complete effacement of the subarachnoid cerebral spinal fluid (CSF) and severe deformity in the cervical medullary junction, abnormal cervical cord T2 signal, and evidence of compressive edema. This patient underwent posterior fossa decompression with $\mathrm{C} 1$ laminectomy 5 days later. At the most recent clinical evaluation at 25 months of age this patient was fully recovered and thriving.

Patient 2 was initially seen at 1 month of age and again at 5 months of age. Physical exam was consistent with achondroplasia. Initial neurological exam was normal and follow-up exam noted hypotonia but no other neurological abnormalities. Medical history was unremarkable. Brain MRI was obtained at 6 months of age and noted severe stenosis at the foramen magnum with complete effacement of the CSF signal both anterior and posterior to the foramen magnum as well as pathologic T2 hyperintensity of the cord at the cervical medullary junction. Patient was evaluated by neurosurgery a week later and had posterior fossa decompression with C1 laminectomy 2 weeks after the MRI. Patient was doing well at her most recent visit at 40 months of age.

\section{RESULTS}

Forty-nine patients had MRI examination of the craniocervical junction, and 27 of these had cord compression. T2 signal changes in the cord were observed in 11 patients, with one additional patient developing signal abnormality on repeat MRI (12 total with signal abnormality). Posterior fossa decompression surgery was performed in 20 (including the patient with signal abnormality on repeat MRI) (Table 1). Surgery was performed on $10 / 15$ patients who had cord compression but no signal abnormality and 10/12 patients with compression with signal abnormality.

Initial physical exam findings were reviewed for all 49 patients. Hypotonia was a common finding and was present in 2 of 11 patients with cord compression and signal abnormality, 6 of 16 patients with cord compression but no signal abnormality, and 3 of 22 patients with no signal abnormality or cord compression. Only 2 of the 27 patients with cord compression on MRI had an initial neurological exam that was abnormal for anything but hypotonia; both had decreased deep-tendon reflexes (DTRs). This was also noted in one patient who did not have cord compression (Table 1).

PSG results were also reviewed for 39 of our patients, specifically focusing on clinically significant central apneic events, defined by standard published criteria as five or more central apneas and/or hypopneas per hour of sleep. ${ }^{26-28}$ Central apnea was detected in $2 / 14$ patients with cord compression and no signal abnormality and none of the nine patients with signal abnormality. One patient who did not have cord compression noted on MRI also had central apnea (Table 1). Desaturations were also tabulated for these patients. Nine of 13 patients with cord compression but no signal abnormality had desaturations $\leq 85 \%$. Six of 10 patients with

Table 1 Findings and treatment of 49 patients with magnetic resonance image (MRI) examinations

\begin{tabular}{llll} 
& Surgery & $\begin{array}{l}\text { Neurological abnormality } \\
\text { (excluding hypotonia) }\end{array}$ & $\begin{array}{l}\text { Central } \\
\text { apnea }\end{array}$ \\
\hline $\begin{array}{l}\text { Cord } \\
\text { compression } \\
(27)\end{array}$ & 20 & $2 / 27$ & $2 / 23$ \\
No & 0 & $1 / 22$ & \\
$\begin{array}{l}\text { compression } \\
(22)\end{array}$ & & & $1 / 16$ \\
\hline
\end{tabular}


signal abnormality had desaturations $\leq 85 \%$, and $8 / 16$ patients who had no cord compression had desaturations on PSG $\leq 85 \%$.

As of January 2017, follow-up data was available for 15/20 patients who underwent surgery (including evaluations by neurosurgery, genetics, MRI) within the last year. Two patients were seen within the last 3 years, one patient within 4 years and two patients were seen within the last 10 and 15 years, respectively. For those 29 patients who did not have posterior fossa decompression, 9 patients were seen in the last year, 5 patients were evaluated within the last 2 years, 5 patients were seen within the last 5 years, 7 patients have been seen within the last 10 years, and 3 patients have been seen within the last 15 years.

\section{DISCUSSION}

In 1995 and 2005 the AAP recommended that neonates with achondroplasia have either CT or MRI to evaluate the size of the foramen magnum (CT) or cervical cord compression (MRI). ${ }^{20,} 21$ More recent consensus recommendations, achieved by a modified Delphi process, recommended that neuroimaging of infants with achondroplasia be limited to those exhibiting abnormal clinical findings (history or physical exam) or polysomnography. ${ }^{22}$ Based on our clinical experience, summarized here, we have concluded, as have others, ${ }^{23}$ that clinical examination and polysomnography alone will not identify a significant number of children with cord compression and/or MRI signal abnormality, and will leave them at risk for central apnea and sudden death.

The two cases described above illustrate the poor sensitivity of the physical exam and history alone for identifying patients with significant cord compression. Patient 1 had only depressed DTRs on exam yet suffered cardiorespiratory arrest 8 days later due to severe cord compression. Patient 2 had no findings aside from hypotonia and had severe cord compression with complete effacement of the CSF signal. PSG was not obtained prior to the cardiorespiratory event and decompression surgery for patient 1, but PSG for patient 2 was obtained 7 days prior to the MRI, and the central apnea index (CAI) was slightly greater than expected for age, but not abnormally elevated.

Our review of 49 patients followed at our institution over nearly 20 years identified only 3 patients with abnormal neurological findings other than hypotonia on physical exam at their initial visits. Hypotonia was observed in children with normal MRIs (3/22) as well as in those with abnormal MRIs $(8 / 27)$. It is well known to be a common finding in infants with achondroplasia ${ }^{29,30}$ and is clearly not predictive of cord injury. Though a rate of $6 \%$ of patients experiencing abnormal neurological symptoms in this cohort falls below the rates published by Pauli et al. in their prospective cohort of 53 patients (abnormal reflexes or clonus present in at least 6.5 to $19.1 \%$ of patients, respectively; total number of patients experiencing any abnormal reflex or clonus not provided in publication), ${ }^{30}$ Hunter et al. reported only $1.6 \%$ of patients under the age of 2 years with cervical neurological signs. ${ }^{31}$
Abnormal reflexes or other neurological signs are indeed worrisome in an infant with achondroplasia; however, we are concerned that there may be risks associated with cord compression that have not yet progressed to hyperreflexia and clonus.

While we agree that "children who are suspected of having symptomatic [foramen magnum stenosis], based on their history or physical exam or surveillance sleep study, should undergo evaluation by MRI," ${ }^{22}$ this approach lacks sensitivity: only $2 / 27$ patients with cord compression had abnormal neurological examinations other than hypotonia and only 2/ 23 patients with cord compression had clinically significant central apnea. A similar experience regarding PSG results was reported by White et al. based on their experience with 17 patients and a review of the literature. ${ }^{24}$ Based on this, these authors did "not recommend polysomnogram as an adequate screening tool for the presence of cervicomedullary spinal cord compression." 24 In total, of the 23 patients with signal abnormality and/or cord compression where PSG data was also available, only 2 had concerning clinical findings or PSG results. Pauli et al. compared PSG desaturations $\leq 85 \%$ in patients who underwent decompression surgery with patients who did not, with the desaturations considered as "evidence for abnormality of central respiratory control." ${ }^{30}$ In our patient cohort, $60 \%$ of patients with signal abnormality on MRI (65\% of all patients with cord compression) compared with $50 \%$ of patients with no cord compression had desaturations that were at or below $85 \%$. Based on our data low desaturations appear to be a poor predictor of the presence of cord compression or signal abnormality. We assert that physical exam findings and history alone are insufficient screening methods to assess which patients with achondroplasia require an MRI and which do not.

A paper from 2014 described the experience at one institution using dynamic MRI of the cervicomedullary junction with cinema CSF flow studies in symptomatic children with achondroplasia. ${ }^{32}$ The authors note that in $4 /$ $29(13.7 \%)$ patients who underwent imaging, cervical cord compression with CSF obliteration was only evident on flexion-extension studies and had not been seen on the MRIs performed in neutral position. This paper raises the question as to whether MRIs done at resting position might be missing some patients with cord compression. This publication presents dynamic MRI findings on patients who are already symptomatic. Because our goal is to screen patients and prevent symptoms before children become symptomatic, we might question whether this should be incorporated into screening. At this time we do not know if cord compression in flexion-extension position is present as an early sign, or is one that develops too late to be useful. This could be an area of future research.

In our patient cohort, $40.8 \%$ of patients underwent cervical junction decompression surgery, which is higher than many published rates of decompression surgery amongst patients with achondroplasia (4.5\% [ref. ${ }^{33}$ ], 6.8\% [ref. ${ }^{31}$ ], 9.4\% [ref. ${ }^{30}$ ], $16 \%$ [ref. ${ }^{34}$ ], 25.9\% [ref. ${ }^{35}$ ]) but still remains consistent 
with the high end of the range reported by some centers (34.6\% [ref. ${ }^{13}$ ], 36.4\% [ref. ${ }^{18}$ ], 42.2\% [ref. ${ }^{36}$ ]). Because of the risk not only of sudden death, reported to range from 0.5 to $7.5 \%$ (refs. ${ }^{5,6,30,33,37}$ ), but also due to the increased risk of additional neurological complications previously discussed, ${ }^{9,11,30}$ our center has a low threshold for referring patients for neurosurgical evaluation following an abnormal MRI. Our intent is not only to prevent death related to cord compression in these patients (our decompression rate is approximately 5 to 15 times higher than most published mortality rates), but also to prevent long-term neurological sequelae related to cord compression, which may not always resolve following decompression surgery. ${ }^{30}$ Because physical exam and PSG are not consistent predictors of how the cervical cord may be affected in an infant with achondroplasia and who may suffer death or other neurological complications, we believe MRI is a necessary tool in the evaluation of children with achondroplasia. Our practice of screening infants by 6 months of age is based on historic discussions with experts in skeletal dysplasias, as well as our experience that some infants (such as patients 1 and 2 in our case examples) may suffer from cord compression much earlier than 12 months of age. If the initial MRI is normal, we rescreen all our patients again at 2 years of age, or sooner should additional concerns warrant earlier rescreening. For the patients who ultimately underwent surgery at our institution, there were no predefined criteria used to determine which patients required surgical intervention, and the decision was made by each respective neurosurgeon. However, all patients did undergo a neurosurgical evaluation prior to surgery, which included a physical exam, review of the MRI, and review of other assessments if available. Furthermore, while we did not review this specifically in this manuscript, not all patients had surgery immediately following their initial neurosurgery evaluation. In some cases, the neurosurgeon requested additional testing, follow-up MRIs, or other follow-up evaluations prior to recommending decompression surgery. Decompression surgeries were performed by four different neurosurgeons in this cohort.

While CT allows for measurement of the size of the foramen magnum and comparison with published standards for infants with achondroplasia ${ }^{38}$ without the need for sedation, CT is unable to demonstrate the impact of stenosis on the cervical spinal cord, which we believe is the advantage of MRI over CT, and which has greater clinical significance for the patient. MRI is not without risks, due to the need for sedation or general anesthesia in infants and children who undergo MRIs. In a retrospective study out of the Netherlands examining 14,300 children undergoing anesthesia for any reason, complications occurred in less than $2.6 \%$ of patients in the group that included anesthesia for MRI (which also included gastrointestinal and bronchoscopic procedures), ${ }^{39}$ and a review by Callahan et al. of anesthesia used in infants and children noted a risk of around 1/10,000 for major morbidity and a $1 \%$ risk of a complication necessitating airway management. ${ }^{40}$ In experienced centers with anesthesia administered by a pediatric anesthesiologist and appropriate monitoring, we believe the risks should be very low.

Young children with achondroplasia are at risk for cervical cord injury and sudden death because of foramen magnum stenosis and cord compression. Prior to the use of MRI to screen patients with achondroplasia for cord compression, sudden death was a small but significant concern for patients, and physicians were limited in the tools available to them to discern who might be at risk. Our data demonstrate that MRI identifies many children with cervical cord compression whose clinical findings and PSG are not worrisome. We recommend that both MRI and PSG be obtained before the age of 6 months in children with achondroplasia, or when the child is first evaluated. If the initial MRI is normal, we recommend repeating the MRI at 2 years of age. Although sedation or general anesthesia is required for MRI at this age, it can be safely performed in centers experienced in the care of young children.

\section{DISCLOSURE}

The authors declare no conflicts of interest.

\section{REFERENCES}

1. Oberklaid F, Danks DM, Jensen F, et al. Achondroplasia and hypochondroplasia. J Med Genet. 1979;16:140-6.

2. Afsharpaiman S, Sillence DO, Sheikhvatan M, et al. Respiratory events and obstructive sleep apnea in children with achondroplasia: investigation and treatment outcomes. Sleep Breath. 2011;15:755-61.

3. Bland JD, Emery JL. Unexpected death of children with achondroplasia after the perinatal period. Dev Med Child Neurol. 1982;24:489-92.

4. Nehme AME, Riseborough EJ, Tredwell SJ. Skeletal growth and development of the achondroplastic dwarf. Clin Orthop. 1976;116:8-23.

5. Hecht JT, Francomano CA, Horton WA, et al. Mortality in achondroplasia. Am J Hum Genet. 1987:41:454-64.

6. Pauli RM, Scott CI, Wassman ER Jr, et al. Apnea and sudden unexpected death in infants with achondroplasia. J Pediatr. 1984;104:342-8.

7. Yang SS, Corbett DP, Brough AJ, et al. Upper cervical myelopathy in achondroplasia. Am J Clin Pathol. 1977;68:68-72.

8. Babiker MOE, Edwards C, Davies $P$, et al. Recurrent acute life-threatening events in a child with achondroplasia. Arch Dis Child Educ Pract Ed. 2015;100:19.

9. Colamaria V, Mazza C, Beltramelloa A, et al. Irreversible respiratory failure in an achondroplastic child: the importance of an early cervicomedullary decompression, and a review of the literature. Brain Dev. 1991;13:270-9.

10. Najjar JA, Peitersen SE, Carter LP. Craniocervical stenosis and apnea spells in a 2-month-old baby with achondroplasia. J Child Neurol. 1995;10:484-6.

11. Cohen ME, Rosenthal AD, Matson DD. Neurological abnormalities in achondroplastic children. J Pediatr. 1967;71:367-76.

12. Benglis DM, Sandberg DI. Acute neurological deficit after minor trauma in an infant with achondroplasia and cervicomedullary compression. J Neurosurg. 2007; 107:152-5.

13. Reid CS, Pyreritz RE, Kopits SE, et al. Cervicomedullary compression in young patients with achondroplasia: value of comprehensive neurologic and respiratory evaluation. J Pediatr. 1987;110:522-30.

14. Fornarino $S$, Rossi DP, Severino $M$, et al. Early impairment of somatosensory evoked potentials in very young children with achondroplasia with foramen magnum stenosis. Dev Med Child Neurol. 2017;59:192-8.

15. Kao SC, Waziri MH, Smith WL, et al. MR imaging of the craniovertebral junction, cranium, and brain in children with achondroplasia. Am J Roentgenol. 1989;153:565-9.

16. Thomas IT, Frias JL, Williams JL, et al. Magnetic resonance imaging in the assessment of medullary compression in achondroplasia. Am J Dis Child. 1988;142:989-92. 
17. Brühl $K$, Stoeter $P$, Wietek $B$, et al. Cerebral spinal fluid flow, venous drainage and spinal cord compression in achondroplastic children: impact of magnetic resonance findings for decompressive surgery at the craniocervical junction. Eur J Pediatr. 2001;160:10-20.

18. Keiper GL Jr, Koch B, Crone KR. Achondroplasia and cervicomedullary compression: prospective evaluation and surgical treatment. Pediatr Neurosurg. 1999;31:78-83.

19. King JAJ, Vachhrajani S, Drake JM, et al. Neurosurgical implications of achondroplasia. J Neurosurg Pediatr. 2009;4:297-306.

20. American Academy of Pediatrics, Committee on Genetics. Health supervision for children with achondroplasia. Pediatrics. 1995;95:443-51.

21. Trotter TL, Hall JG, American Academy of Pediatrics, Committee on Genetics. Health supervision for children with achondroplasia. Pediatrics. 2005;116:771-83.

22. White KK, Bompadre V, Goldberg MJ, et al. Best practices in the evaluation and treatment of foramen magnum stenosis in achondroplasia during infancy. Am J Med Genet A. 2016;170A:42-51.

23. Pauli RM. Letter to the editor: response to two recent articles regarding achondroplasia. Am J Med Genet A. 2016;170A:1099-100.

24. White KK, Parnell SE, Kifle $Y$, et al. Is there a correlation between sleep disordered breathing and foramen magnum stenosis in children with achondroplasia? Am J Med Genet A. 2016;170A:32-41.

25. White KK, Savarirayan R, Goldberg MJ, et al. Response: "Best practices in the evaluation and treatment of foramen magnum stenosis in achondroplasia during infancy" and "Is there a correlation between sleep disordered breathing and foramen magnum stenosis in children with achondroplasia?". Am J Med Genet A. 2016;170A:1101-3.

26. Anders $T$, Emde $R$, Parmelee A, editors. A manual of standardized terminology, techniques and criteria for scoring of states of sleep and wakefulness in newborn infants. Los Angeles: UCLA Brain Information Service, 1971.

27. Crowell DH, CHIME Study Group. An atlas of infant polysomnography. New York: Parthenon Publishing Group; 2003. p. 168.

28. Sheldon SH. Evaluating sleep in infants and children. Philadelphia: Lippincott-Raven; 1996. p. 276.

29. Ruiz-Garcia M, Tovar-Baudin A, Del Castillo-Ruiz V, et al. Early detection of neurological manifestations in achondroplasia. Childs Nerv Syst. 1997;13:208-13.
30. Pauli RM, Horton VK, Glinski LP, et al. Prospective assessment of risks for cervicomedullary-junction compression in infants with achondroplasia. Am J Hum Genet. 1995;56:732-44.

31. Hunter AGW, Bankier A, Rogers JG, et al. Medical complications of achondroplasia: a multicentre patient review. J Med Genet. 1998;35:705-12.

32. Mukerjee D, Pressman BD, Krakow D, et al. Dynamic cervicomedullary cord compression and alterations in cerebrospinal fluid dynamics in children with achondroplasia: review of an 11-year surgical case series. J Neurosurg Pediatr. 2014;14:238-44.

33. Rimoin DL. Cervicomedullary junction compression in infants with achondroplasia: when to perform neurosurgical decompression. Am J Hum Genet. 1995;56:824-7.

34. Ireland PJ, Johnson S, Donaghey S, et al. Medical management of children with achondroplasia: evaluation of an Australasian cohort aged 0-5 years. J Paediatr Child Health. 2012;48:443-9.

35. Aryanpur J, Hurko O, Francomano C, et al. Craniocervical decompression for cervicomedullary compression in pediatric patients with achondroplasia. J Neurosurg. 1990;73:375-82.

36. Francomano CA, Carson B, Seidler A, et al. Morbidity and mortality in achondroplasia: efficacy of prospective evaluation and surgical intervention. Am J Hum Genet Suppl. 1993;53:A112.

37. Simmons K, Hashmi SS, Scheuerle A, et al. Mortality in babies with achondroplasia: revisited. Birth Defects Res A Clin Mol Teratol. 2014;100:247-9.

38. Hecht JT, Horton WA, Reid CS, et al. Growth of the foramen magnum in achondroplasia. Am J Med Genet. 1989;32:528-35.

39. Westerkamp AC, de Geus AF, Molenbuur B, et al. Comparing perioperative complications of paediatric and adult anaesthesia: a retrospective cohort study of 81267 cases. Eur J Anaesthesiol. 2018;35:280-8.

40. Callahan MJ, MacDougall RD, Bixby SD, et al. Ionizing radiation from computed tomography versus anesthesia for magnetic resonance imaging in infants and children: patient safety considerations. Pediatr Radiol. 2018;48:21-30. 